\title{
Citizens, research ethics committee members and researchers' attitude toward information and consent for the secondary use of health data: Implications for research within learning health systems
}

\author{
Annabelle Cumyn ${ }^{1,2}$, M.D,C.M, MHPE \\ Roxanne Dault ${ }^{1,4}, \mathrm{MSC}$ \\ Adrien Barton $^{1,3}, \mathrm{PhD}$ \\ Anne-Marie Cloutier ${ }^{1,4}$, MSc \\ Jean-François Ethier ${ }^{1,2,4}$, M.D,C.M PhD
}

1- Groupe de recherche interdisciplinaire en informatique de la santé (GRIIS), Faculté de médecine et des sciences de la santé/Faculté des sciences, Université de Sherbrooke, Québec, Canada.

2- Département de médecine, Faculté de médecine et des sciences de la santé, Université de Sherbrooke, Québec, Canada.

3- Centre national de la recherche scientifique - Institut de recherche en informatique de Toulouse (CNRS-IRIT), Toulouse, France.

4- Data Access component, QuebecSPOR Support Unit, Université de Sherbrooke, Québec, Canada.

\section{Corresponding author}

Jean-François Ethier,

Groupe de recherche interdisciplinaire en informatique de la santé (GRIIS)

Faculté de Médecine et des Sciences de la santé / Faculté des Sciences

Université de Sherbrooke,

Phone: 819-346-1110, x74977

email: Jean-Francois.Ethier@USherbrooke.ca 


\begin{abstract}
A provincial survey was conducted to assess citizens, research ethics committee (REC) members and researchers' attitude toward information and consent for the secondary use of health data for research within a learning health system (LHS). The results show that: 1) reuse of health data for research to advance knowledge and improve care is values by all parties; 2 ) consent regarding health data use for research is fundamental particularly to citizens, even when the data are deidentified; 3 ) a secure website to support the information and consent processes was appreciated by all. Researchers were more comfortable with the use of de-identified health data for research without informing nor seeking consent from people than citizen and REC member respondents. This survey was part of a larger project that aims at exploring public perspectives on alternate approaches to the current consent models in Quebec to take into consideration the unique features of LHS. The revised consent model will need to ensure that citizens are given the opportunity to be better informed about incoming researches with their health data and have their say, when possible, in the use of their data.
\end{abstract}

\title{
Keywords
}

health data, secondary use, learning health systems, survey, research ethics, informed consent 


\section{Introduction}

In the last decade, implementations of learning healthcare systems (LHSs) are on the rise around the world (Lessard et al., 2017). LHSs focus on closely coupling clinical care with both the conduct of research and the translation of research into practice. Ensuing changes to care practice offera new start for the next iteration of the cycle. Their development reflects the intention to use, on a large scale, people's health data generated on a daily basis within the healthcare system to facilitate the research and clinical continuum (Delaney et al., 2013; The learning healthcare project, 2020). For example, LHSs aim to improve healthcare by allowing personalised models of care. They may also reduce the pressure on the healthcare system and its costs (Budrionis \& Bellika, 2016). The endpoint is to fulfill the promise of evidence-based medicine by: 1) enabling a research pertinent to an organisation or jurisdiction that rapidly generates new knowledge that can be swiftly applied in current clinical practice (changing the focus from research projects to learning cycle), 2) introducing a culture of shared responsibilities, and 3) facilitating the participation of citizens, patients and healthcare providers in the production and dissemination of new knowledge (Budrionis \& Bellika, 2016).

The province of Quebec (Canada) is currently working on the implementation of a learning health data platform named the "Learning Health and Social Services Research Platform" (Plateforme apprenante pour la recherche en santé et services sociaux au Québec in French, abbreviated "PARS3") to support the secondary use of primary care health data in research as well as for other LHS activities, such as reflective practice and quality improvement (GRIIS, 2019). This platform will therefore enable (with proper authorisations, obviously) a more complete understanding of an individual, through her/his health data. While obvious sources include hospitals' and clinics' electronic health records, other important sources include research repositories or connected devices. This implies a shift of focus by putting the individual (rather than an organisation) at the center of the system. This poses significant challenges as all these sources use heterogenous technological systems. To solve this aspect, PARS3 relies on a clinical ontological model supporting semantic interoperability across various data systems (GRIIS, 2019). Access to this data will be highly regulated according to information security standards, and provision of the necessary access permissions such as REC approval for research.

In Canada, federal laws protect personal data and each province is responsible for governance of healthcare. In the province of Quebec, access to hospital-based health data is regulated by the 
Act respecting health services and social services ${ }^{1}$ and consent to participation in research by the Civil Code of Quebec ${ }^{2}$. Research in all Canadian institutions funded by three federal agencies must comply with the Tri-Council Policy Statement: Ethical Conduct for Research Involving humans (Government of Canada, 2019). Canadian and provincial normative and legal frameworks were however not established with learning platforms in mind. Several authors beyond the Canadian context have argued that current consent models are not optimal for LHSs nor do they take into account the aspects inherent to LHSs like the requirement to go through knowledge transfer as part of the cycle, thereby increasing the likelihood of transformative impact on care (R. R. Faden et al., 2013). The current standard of project-specific individual consent is inoperable on such a scale and would not enable to reach the social benefits of data reuse. Delegated consent for access to hospital-based health data, possible in specific contexts under the Act respecting health services and social services ${ }^{3}$, lacks provisions permitting healthcare users to be informed about the use of their health data and express their consent or dissent regarding their use for research or other purposes.

Thus, a major consideration in the implementation of a LHS is how to inform and support consent from individuals for the reuse of their health data for research with an approach that balances patient autonomy, transparency and social benefits of data reuse. An exhaustive literature review performed in 2018 allowed us to analyse characteristics of different consent models that could be implemented within a LHS (Cumyn et al., 2019). It identified the meta-consent model proposed by Ploug \& Holm (2016) as a very promising model. This model aims to be flexible by offering options for specific, broad or blanket consent (or symmetric options for refusal), combined with dynamic consent (Ploug \& Holm, 2016). The model has been proposed to answer the challenges of informed consent and registry-based or biobank research (Ploug \& Holm, 2017).

With the start of the implementation of a LHS platform in Quebec, we were particularly interested to survey the opinions of concerned parties, and specifically citizens, regarding information and consent for the secondary use of health data for research. The objective of this study was to conduct a province-wide survey to examine Quebec citizens, research ethics committee (REC) members and researchers' points of view about the secondary use of health data in research,

\footnotetext{
${ }^{1}$ Act respecting health services and social services, CQLR, c S-4.2.

${ }^{2}$ Civil Code of Quebec, CQLR, c CCQ-1991, s 20.

${ }^{3}$ Act respecting health services and social services, CQLR, c S-4.2.
} 
including the importance of information and consent as well as their attitude toward fundamental aspects of a new model of consent (meta-consent) adapted to LHSs. This survey was part of a mixed-method study to inform a series of focus groups to deepen our understanding of Quebec citizens' opinion about the meta-consent model for the reuse of their health data for research.

\section{Method}

\section{Sampling strategy and population}

\section{Citizens}

A group of 50,000 phone numbers was randomly generated from a sampling frame owned by BIP Research polling firm, which contained 1,947,000 phone numbers and postal codes from the entire province of Quebec (BIP Research, 2015). This group consists of 75\% landline numbers and $25 \%$ cell phone numbers. Using the six-digit postal code, the random group was then stratified by administrative region in order to represent the entire Quebec territory. Quota were also used for gender, age and education to ensure inclusion of each of these key population subgroups in the sample. Only adults ( $\geq 18$ years old) and residents of the province of Quebec were eligible to participate in the survey. For landline numbers, only one adult per household was asked to take part in the survey. As for cell phone numbers, the holder of the phone line was asked to participate, if eligible.

From this group, the aim was to obtain 385 respondents. Our primary goal was to estimate characteristics of the general population. Classically, using a normal distribution of answers, a margin of error of 5\%, a confidence interval of $95 \%$ (z-score of 1.96) and assuming a standard deviation of 0.5 for a population of 8.38 million persons (Quebec, 2018), the suggested sample size is 385. Nevertheless, the questions are answered using a 5-points Likert scale and so these assumptions are unlikely to hold. This context has been evaluated before, a method has been proposed by Park \& Jung (2009) and has been used in a context very similar to ours (Karbwang et al., 2018). This method suggests that assumptions from a traditional estimation sample size overestimate the sampling requirement in context of answers given using a Likert scale and that a sample size of 231 would allow a margin of error of $5 \%$ given the estimated parameters. Nevertheless, given a certain dose of uncertainty inherent to the estimation of parameters a priori, we decided to aim for the worst-case scenario and target 385 respondents.

Researchers and REC members 
A convenience sampling method was used for researchers and REC members. Directors from 19 research centers and coordinators from 33 network RECs across the province of Quebec were contacted by our research team. Directors and coordinators, identified by a publicly available online list (Gouvernement du Québec, 2020a, 2020b), were asked to share an invitation to participate in an online version of the survey to their researchers and REC members. Overall, 8 research centers (42\%) and 22 network RECs $(67 \%)$ accepted to share the invitation to their teams. Only researchers who have been primary investigator or co-investigator on a research project requiring the approval of a human REC were eligible to participate in the survey. The survey was programmed and self-administered online using the LimeSurvey software version 3.21.3. Multiple survey completions were avoided by use of a unique URLfor each survey. Data collection was anonymous.

\section{Survey development and content}

The survey content was developed from themes previously identified in our scoping review (Cumyn et al., 2019). Although some prior research used scenario-based questionnaires, upon consultation with experts in literacy, we chose a statement-based questionnaire that could be administered by phone and that would inform subsequent focus groups. Supported by an expert in methodology, the research team developed a 13-items survey using closed-ended statements. The full survey, as administered to the respondents, is presented in a supplemental file (Appendix 1). For the first twelve statements (S1-S12), respondents were asked to indicate their level of agreement on a 5-points Likert scale ("strongly agree", "somewhat agree", "neither agree nor disagree", "somewhat disagree", "strongly disagree"). For the last statement (S13), respondents had to choose between two statements, whichever one they considered most important to them.

The 13-items survey covered five main domains investigating citizens, researchers and REC members' attitude toward:

- the secondary use of people's health data in research (S1). In this survey, the definition of health data provided to respondents was information included in an electronic medical record such as any health diagnoses, test results, doctor and nurse notes, as well as drug prescriptions. Research with health data was defined as research that reuses already existing health information. We excluded clinical and prospective research from this definition. 
- the level of information and consent acceptable regarding the use of de-identified health data and identifying health data in research (S2-S7). In this survey, identifying health data was defined as data that contains information that could permit direct identification (e.g. a postal address). This definition was chosen to reflect the occasional research project where health data needs to be coupled with environmental data, for example.

- Scenario 1. Information is provided regarding the use of people's health data and consent is requested.

- Scenario 2. Information is provided regarding the use of people's health data, but consent is not requested.

- Scenario 3. No information is provided regarding the use of people's health data and consent is not requested.

- Characteristics of a meta-consent model(S8-S10):

- the acceptability of the implementation of a secure website to allow citizens to be informed and to express their consent preferences for the secondary use of their health data for upcoming research projects;

- the importance of support from health professionals for the use of the secure website;

- the acceptability of default settings that allow the use of health data according to current laws when people did not express their consent preferences.

- Delegation of consent to a third party (S11-S12):

- the delegation of consent to a director of professional services (current situation in Quebec for the secondary use of hospital health data for research);

- the delegation of consent to RECs (hypothetical situation).

- Relative valuation of individual control vs. the societal benefits of research (S13).

\section{Survey testing and administration}

\section{Citizens}

The survey was administered to Quebec citizens by BIP Research, a professional polling firm, between June 27, 2018 and July 7, 2018 (BIP Research, 2015). The survey was administered by phone by experienced interviewers previously trained for the purpose of this study. The interviews were conducted in both French and English. Prior to the official data collection, two recorded pretests were conducted with $10 \%$ of the sample in order to adjust the survey content and to standardise the administration between interviewers. These interviews were discarded. 


\section{Researchers and REC members}

The web-based survey was administered to researchers and REC members between October 22, 2019 and December 20, 2019. During data collection period, two reminders were sent to increase the response rate.

Two different methods of data collection were used respectively for citizens and researchers/REC members. For citizens, a phone administration was preferred over an online administration in order to promote the inclusion of people with a low literacy level (e.g., difficulties with reading). As for researchers and REC members, we opted for an online administration because it was the easiest way to reach these populations. Minor modifications were made between the phone and the web-based versions of the survey to adapt their respective formats. However, the content of the survey was identical between groups in order to avoid changing the meaning. Tests were made to ensure that a similar level of comprehension of survey content was obtained from the two administration methods.

\section{Statistical analysis}

Frequencies were used to describe the respondent characteristics and responses to each items of the survey. Citizen characteristics were compared with those of the entire Quebec population using chi-squared tests.

The difference in the point of view between the three groups (citizens, REC members and researchers) was also assessed for each of the survey items. First, responses to each statement were pooled into three categories: agreement ("strongly agree" and "somewhat agree"), neutral ("neither agree nor disagree") and disagreement ("strongly disagree" and "somewhat disagree"). Then, the difference in level of agreement between the three groups was statistically compared using chi-squared tests or Fisher's exact tests. Two-tailed tests were used, and $p$-values of 0.05 or less were considered significant. Analyses were performed using SPSS IBM Statistics version 26.

\section{Results}

\section{Citizens}

The professional pooling firm used a random selection of 1,961 phone numbers and finally yielded a sample size of 387 Quebec adult citizen respondents. The response rate was $20 \%$ whereas the cooperation rate (rate of reached individuals who agreed to participate in the survey) was $38 \%$. These percentages are similar to the ones observed in national US random-digit dialed surveys 
(Fox, 2011; Kim et al., 2015). The interviews took an average of 14.3 minutes with a standard deviation of 2.66 minutes.

Researchers and REC members

For the web-based survey, 99 REC members and 66 researchers completed the questionnaire. The completion rate, namely the rate of respondents who completed questionnaires among those who attempted to complete it, was $75 \%$ (165 of 220 respondents). Among the 55 people who did not completed the survey, 29 are unidentified (they did not answer the first question in the questionnaire related to identification ), 17 are researchers and 9 are REC members.

\section{Characteristics of the respondents}

Table 1 compares survey citizen respondents with the general Quebec population. Our respondents differed significantly from the 2018 statistics of the Quebec population as reported by the Institut de la Statistique du Québec (Institut de la Statistique du Québec, 2019; Institut de la statistique du Québec, 2018) in three aspects: age, level of education and language. Our sample had a lower proportion of young people (18-44 years old: $28.2 \%$ vs $41.7 \%$ ) and a higher proportion of elderly (65 years old and more: $34.1 \%$ vs $22.7 \%$ ), - which is often the case for surveys, as well as a lower proportion of individuals with no high school diploma ( $8.5 \%$ vs. $11.3 \%$ ). In addition, there were more native French speakers in our sample compared with the entire population of Quebec (90.4\% vs 78.1\%) even if the survey was administered in both French and English. All administrative regions of the Quebec province were represented in the survey, including both rural and urban areas (information not presented in Table 1). Most of the respondents never took part in a health research study (82.4\%) and slightly more than half visited a hospital or a medical clinic three times or more in the last yearfor themselves or for a loved one.

Table 1. Characteristics of the citizen respondents compared to the population of the province of Quebec (Canada).

\begin{tabular}{|c|c|c|c|}
\hline & $\begin{array}{l}\text { Respondents } \\
\quad(n=387)\end{array}$ & $\begin{array}{c}\text { Quebec } \\
\text { population* }\end{array}$ & $p$ \\
\hline \multicolumn{4}{|l|}{ Gender } \\
\hline Male & $47.5 \%$ & $49.2 \%$ & 0.515 \\
\hline Female & $52.5 \%$ & $50.8 \%$ & \\
\hline \multicolumn{4}{|l|}{ Age } \\
\hline $18-44$ years old & $28.2 \%$ & $41.7 \%$ & $<0.001$ \\
\hline $45-64$ years old & $28.4 \%$ & $35.5 \%$ & \\
\hline 65 years old and more & $34.1 \%$ & $22.7 \%$ & \\
\hline \multicolumn{4}{|l|}{ Higher diploma obtained } \\
\hline No diploma & $8.5 \%$ & $11.3 \%$ & $<0.001$ \\
\hline
\end{tabular}




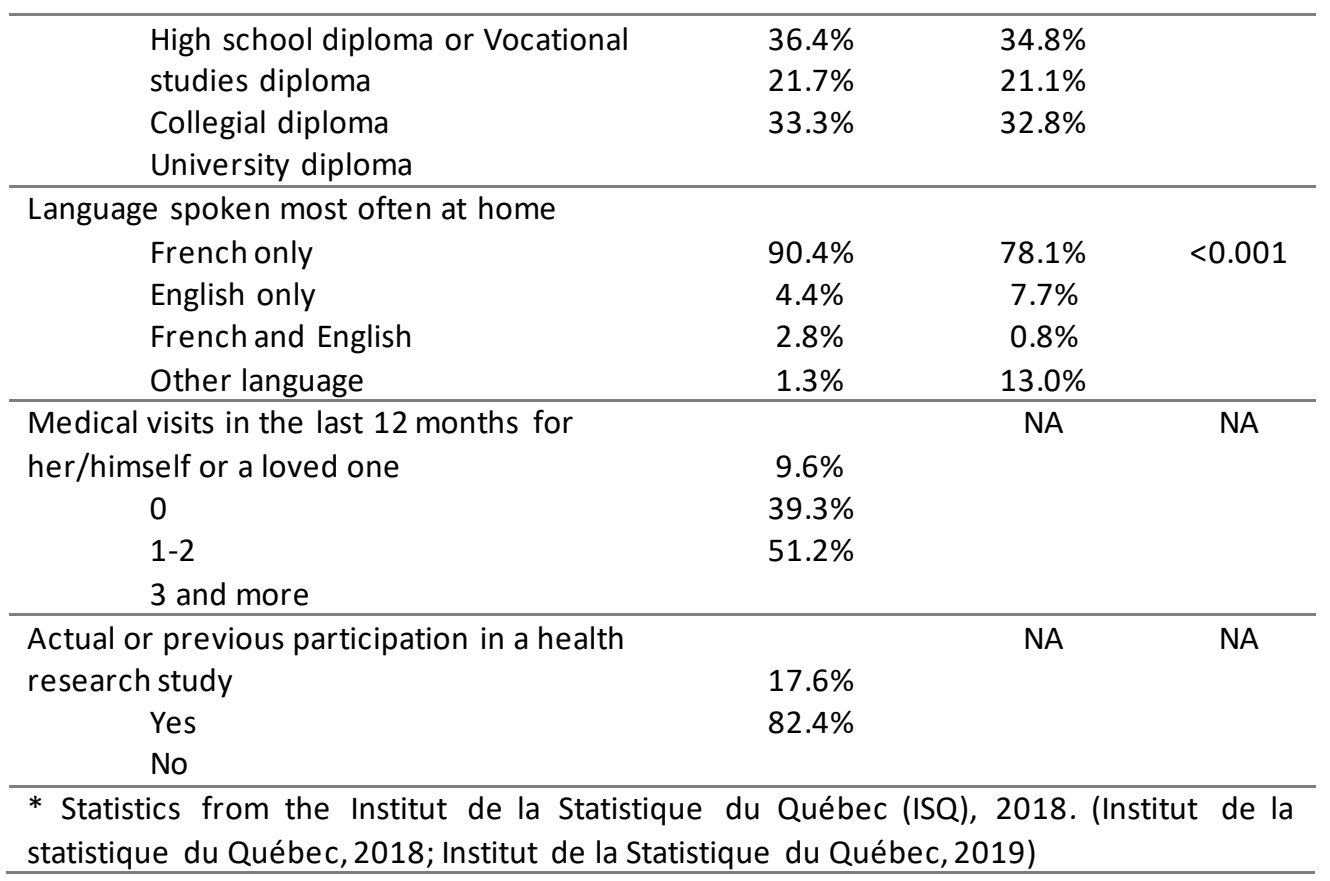

Table 2 presents the characteristics of the REC members and the researchers. $38 \%$ of REC member respondents have a scientific role in their committee, $26 \%$ are public representatives and $16 \%$ are ethicists. Health professionals, legal experts, coordinators as well as students were also represented in the sample. Close to $80 \%$ of them have two or more years of expe rience as active members of a REC. As for researchers, $83.3 \%$ have already used health data for their research projects as a principal investigator or a co-investigator.

Table 2. Characteristics of the REC member and the researcher respondents.

\begin{tabular}{|c|c|c|c|}
\hline & $\begin{array}{c}\text { Total } \\
(n=165)\end{array}$ & $\begin{array}{l}\text { REC members } \\
\qquad(n=99)\end{array}$ & $\begin{array}{l}\text { Researchers } \\
\quad(n=66)\end{array}$ \\
\hline \multicolumn{4}{|l|}{ Gender } \\
\hline Male & $39.4 \%$ & $38.4 \%$ & $40.9 \%$ \\
\hline Female & $54.0 \%$ & $56.6 \%$ & $50.0 \%$ \\
\hline Not want to answer & $6.7 \%$ & $5.1 \%$ & $9.1 \%$ \\
\hline \multicolumn{4}{|l|}{ Age } \\
\hline $18-44$ years old & $38.2 \%$ & $39.4 \%$ & $36.3 \%$ \\
\hline $45-64$ years old & $43.6 \%$ & $38.4 \%$ & $51.5 \%$ \\
\hline 65 years old and more & $13.3 \%$ & $18.2 \%$ & $6.1 \%$ \\
\hline Not want to answer & $4.8 \%$ & $4.0 \%$ & $6.1 \%$ \\
\hline $\begin{array}{l}\text { Uses of health data for a research } \\
\text { project }\end{array}$ & NA & NA & \\
\hline Yes & & & $83.3 \%$ \\
\hline No & & & $16.7 \%$ \\
\hline Role in the REC & NA & & NA \\
\hline Scientist & & $38.4 \%$ & \\
\hline Citizen & & $26.3 \%$ & \\
\hline
\end{tabular}




\begin{tabular}{lc}
\hline Ethicist & $16.2 \%$ \\
Health professional & $7.1 \%$ \\
Legal expert & $5.1 \%$ \\
Coordinator & $4.0 \%$ \\
Student & $1.0 \%$ \\
Dual role & $2.0 \%$ \\
\hline Year of experience as a member of a REC & NA \\
Less than 2 years & $22.2 \%$ \\
2 years and more & $77.8 \%$ \\
\hline REC: Research Ethics Committee. & \\
\hline
\end{tabular}

What are the respondents' opinions regarding the secondary use of people's health data for research?

Citizens were mostly supportive of the secondary use of people's health data for research to advance medical knowledge and improve patient care; $61.2 \%$ strongly agreed and $31.0 \%$ somewhat agreed with this statement while only few respondents were neutral or disagreed (3.1\% neither agreed nor disagreed, 1.3\% somewhat disagreed and 3.4\% strongly disagreed) (Figure 1). REC members had a similar level of agreement, but were more evenly split between strongly and somewhat agreed (49.5\% strongly agreed and $43.4 \%$ somewhat agreed with the statement) (Figure 2) while researchers showed a strong support ( $89.4 \%$ strongly agreed and $6.1 \%$ somewhat agreed with the statement) (Figure 3). Overall, all respondents surveyed were supportive of the general reuse of people's health data for research purposes to advance knowledge and improve care.

\section{What are the respondents' opinions regarding the level of information and consent for the secondary use of de-identified and identifying health data for research?}

Scenario 1. Information is provided and consent is requested: Citizens considered acceptable to use people's health data for research, whether they are de-identified or identifying, if people are informed about the use of their data and asked for their consent (respectively, $91.7 \%$ and $90.4 \%$ strongly or somewhat agreed with this statement) (Figure 1). REC members and researchers seemed to share a similar opinion to citizens, with however, slightly higher proportions of respondents who are strongly in agreement with these statements (Figures 2 and 3). There was no statistical difference between the three groups of respondents for both de-identified or identifying data ( $p=0.138$ and $p=0.373$, respectively) (Table 3 ).

Scenario 2. Information is provided but consent is not requested: For de-identified data, citizens gave polarised answers ( $22.2 \%$ strongly agreed, $26.4 \%$ somewhat agreed, $6.2 \%$ neither agreed nor 
disagreed, $25.8 \%$ somewhat disagreed and $19.4 \%$ strongly disagreed) (Figure 1). However, when identifying data were concerned, citizens mainly disagreed with this statement (36.2\% somewhat disagreed and $28.4 \%$ strongly disagreed). The opinion of REC members regarding de-identified data and identifying data in this context was very similar to citizens' opinion (Figure 2). However, it appears that a larger proportion of researchers agreed (strongly or somewhat agreed) that people's de-identified or identifying health data could be used for research without their consent if they are informed about the use of their data (Figure 3). The differences in the proportions between the three groups of respondents were statistically significant: Researchers seemed more comfortable, compared to citizens and REC members, to use people's de-identified or identifying health data for research purposes when information is provided regarding data use, but consent is not sought ( $p<0.001$ for both de-identified and identifying data) (Table 3 ).

Scenario 3. No information is provided, and consent is not requested: For this third scenario, the level of agreement of each group regarding de-identified health data was similar with the previous scenario (Figures 1, 2 and 3). In the case of identifying health data, acceptance across the three groups of respondents surveyed was low. It appears, however, that the difference in the level of agreement between the three groups is statistically significant: Again, researchers seemed more comfortable, compared to citizens and REC members, to use people's de-identified or identifying health data for research purposes without informing them and without seeking their consent ( $p<0.001$ for both de-identified and identifying data) (Table 3 ).

\section{What are the respondents' opinions toward certain aspects of the meta-consent model?}

Creation and accessibility of a secure website to allow citizens to be informed and to express their consent preferences regarding the use of their health data for upcoming research projects: Close to $80 \%$ of citizens showed a positive attitude for the creation of such a secure website $(50.4 \%$ strongly agreed and $29.5 \%$ somewhat agreed with this statement). Interestingly, REC members appeared to be less motivated than citizens towards such a website ( $36.4 \%$ strongly agreed and $37.4 \%$ somewhat agreed) and researchers showed a similar enthusiasm to that of citizens ( $51.5 \%$ strongly agreed and $19.7 \%$ somewhat agreed). However, there was no statistical differences between the three groups of respondents $(p=0.171)$.

Support from health professionals for the use of the secure website: A great majority of citizens (58.4\% strongly agreed and $29.2 \%$ somewhat agreed) were in favor of health professionals providing support to patients for the use of the website, including help to express consent 
preferences. A great majority of REC members and researchers were also in agreement with this statement (Figures 2 and 3). However, it appeared to be significantly more important for citizens ( $87.6 \%$ of citizens strongly or somewhat agreed vs. $75.8 \%$ of REC members vs. $78.8 \%$ of researchers, $p=0.006$ ) (Table 3).

Acceptability of default settings on the secure website: We asked the respondents if it was acceptable to use people's health data for research if they did not express their consent preferences regarding the use of their data, as long as Quebec's health data access regulations were satisfied. This statement was intended in part to test the acceptability of current regulations permitting retrospective research with record-based health data. In addition, a better understanding of citizen expectations would help to define default settings on the secure website. The presence of default settings corresponds to one of the six essential elements of a metaconsent model (Ploug \& Holm, 2016). There was a strong divergence between the three groups of respondents regarding this statement. $64.6 \%$ of citizen respondents strongly or somewhat agreed with this statement. Only $44.4 \%$ of REC members agreed $(20.2 \%$ strongly agreed and $24.2 \%$ somewhat agreed) with the existence of default settings other than "no" while on the contrary, a strong majority of researchers support it ( $56.1 \%$ strongly agreed and $27.3 \%$ somewhat agreed with this statement). The difference between the three groups was statistically significant $(p<0.001)$ (Table 3).

\section{What are the respondents' opinions toward delegation of consent to a third party?}

In the last set of statements, we presented two different scenarios in which we measured the level of agreement about delegation of consent to a third party for the use of people's health data for research. In the first statement, we evaluated the acceptability to delegate consent to a member of the hospital administration (director of professional services), which is allowed in Quebec for retrospective research with hospital health data, while in the second statement, we evaluated the acceptability to delegate consent to RECs. Only one-third of citizens were in agreement ( $14.0 \%$ strongly agreed and $18.9 \%$ somewhat agreed) with the delegation of consent to a director of professional services (that is, with the current regulation). Half of citizens agreed with the hypothetical scenario, which is to delegate consent to RECs (20.2\% strongly agreed and $30.0 \%$ somewhat agreed) (Figure 1). It appears that the current situation in the province of Quebec whereby a member of the hospital administration may grant authorisation to access hospital-based health data for research is similarly not well accepted by the other respondents 
(Figures 2 and 3). There was no general agreement from REC members regarding REC's hypothetical decisional power to decide, on people's behalf, the use of their health data for research purposes (19.2\% strongly agreed, $26.3 \%$ somewhat agreed, $15.2 \%$ neither agreed nor disagreed, 20.2\% somewhat disagreed and $19.2 \%$ strongly disagreed) (Figure 2). On the other hand, a majority of researchers would support this hypothetical scenario (48.5\% strongly agreed and $28.8 \%$ somewhat agreed with this statement) (Figure 3 ). The difference observed between the three groups of respondents was statistically significant $(p<0.001)$ (Table 3).

\section{Which value, between individual control on personal data and common good through medical} research, is the most important for the respondents?

For the last part of the survey, we asked the respondents to choose between two statements that intended to evaluate which value was the most important for them between individual control on their data (which is related to autonomy) and fostering common good through an easier medical research. Citizens expressed more agreement with the statement relating to individual control $(61.5 \%)$ than the one relating to common good (37.5\%), suggesting that they value their individual control more than the facilitation of research (Table 4). Like citizens, the majority of REC members expressed more agreement with the statement relating to individual control (63.6\%). On the contrary, researchers expressed more agreement with the statement relating to common good (69.7\%). 


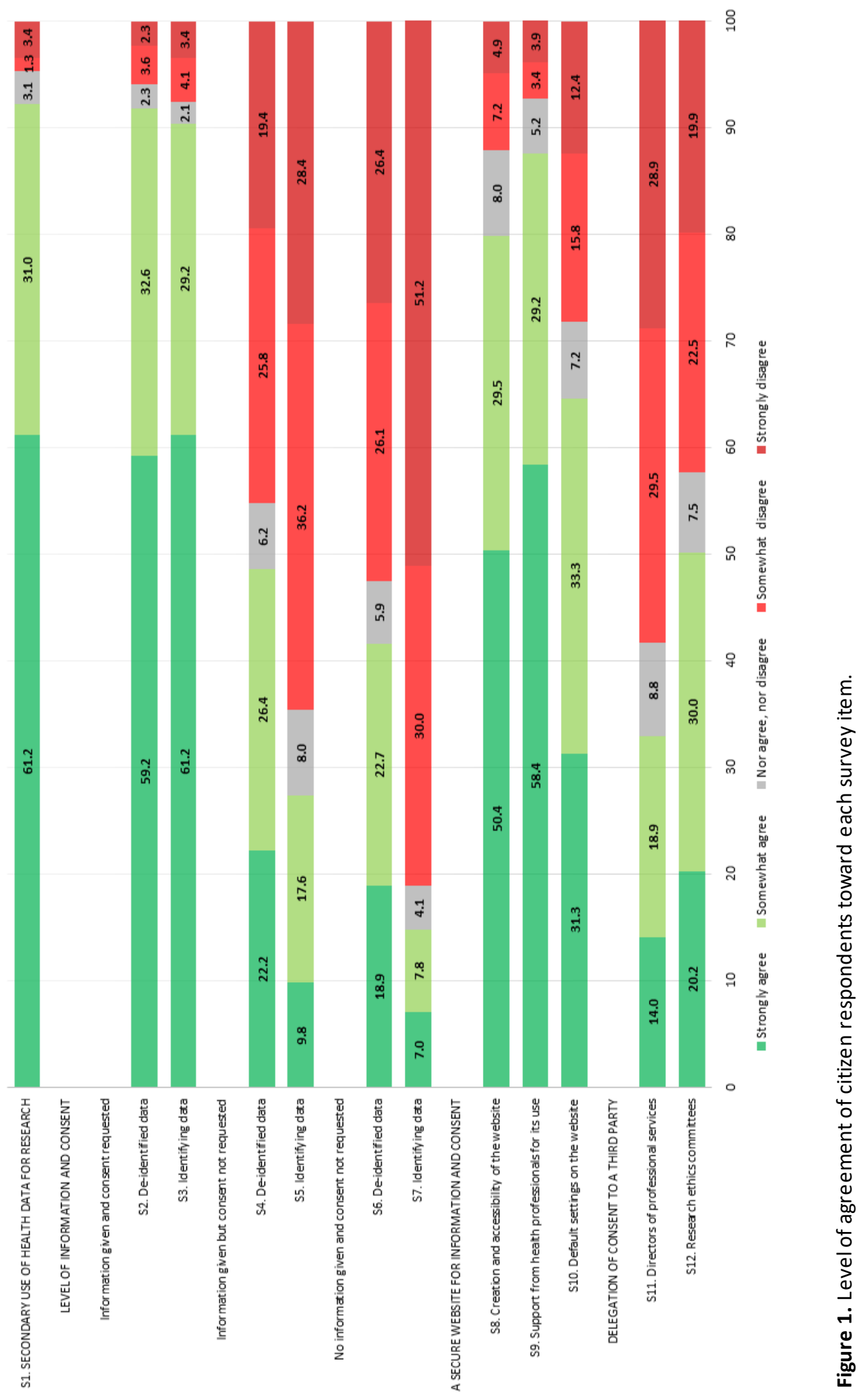




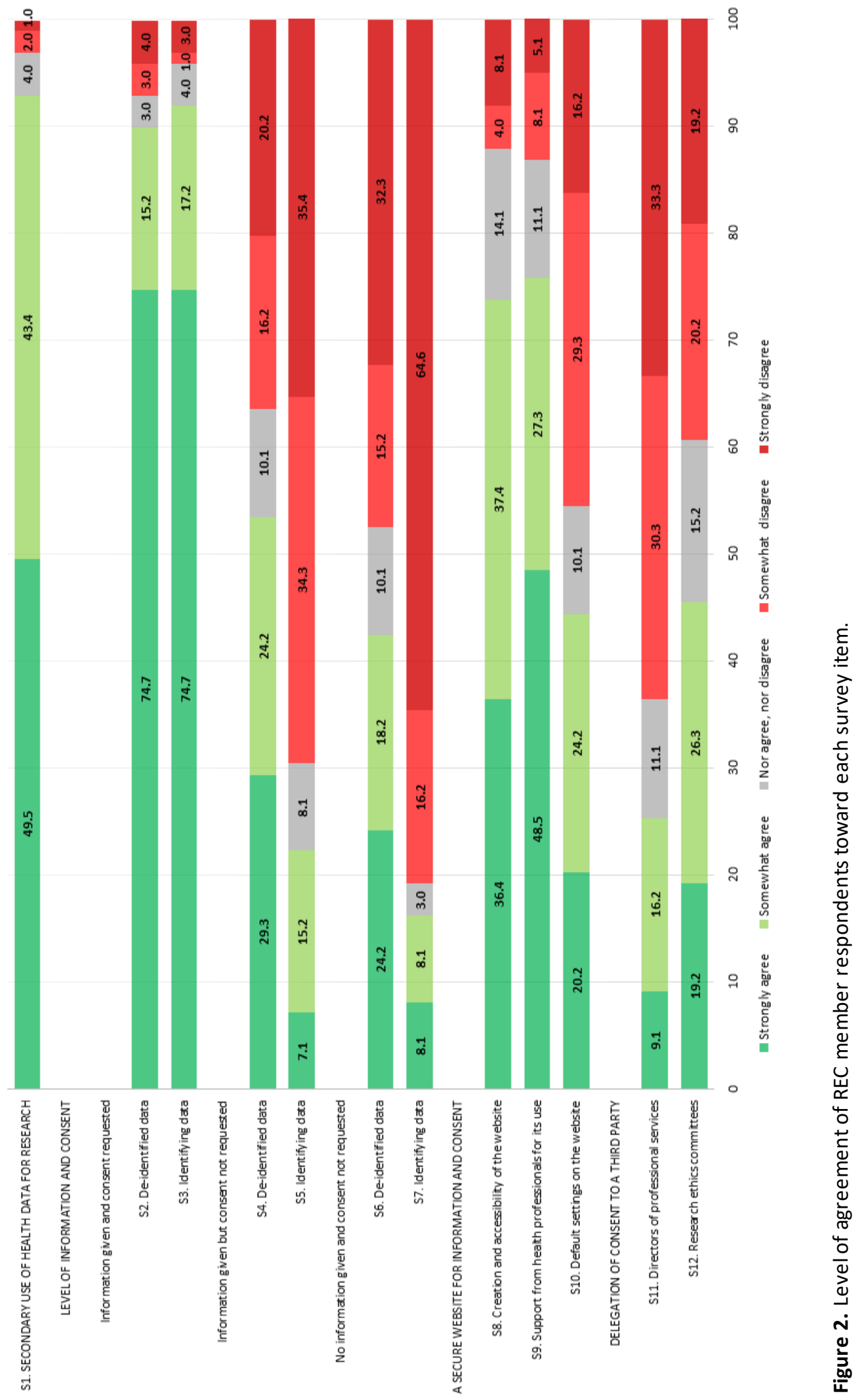




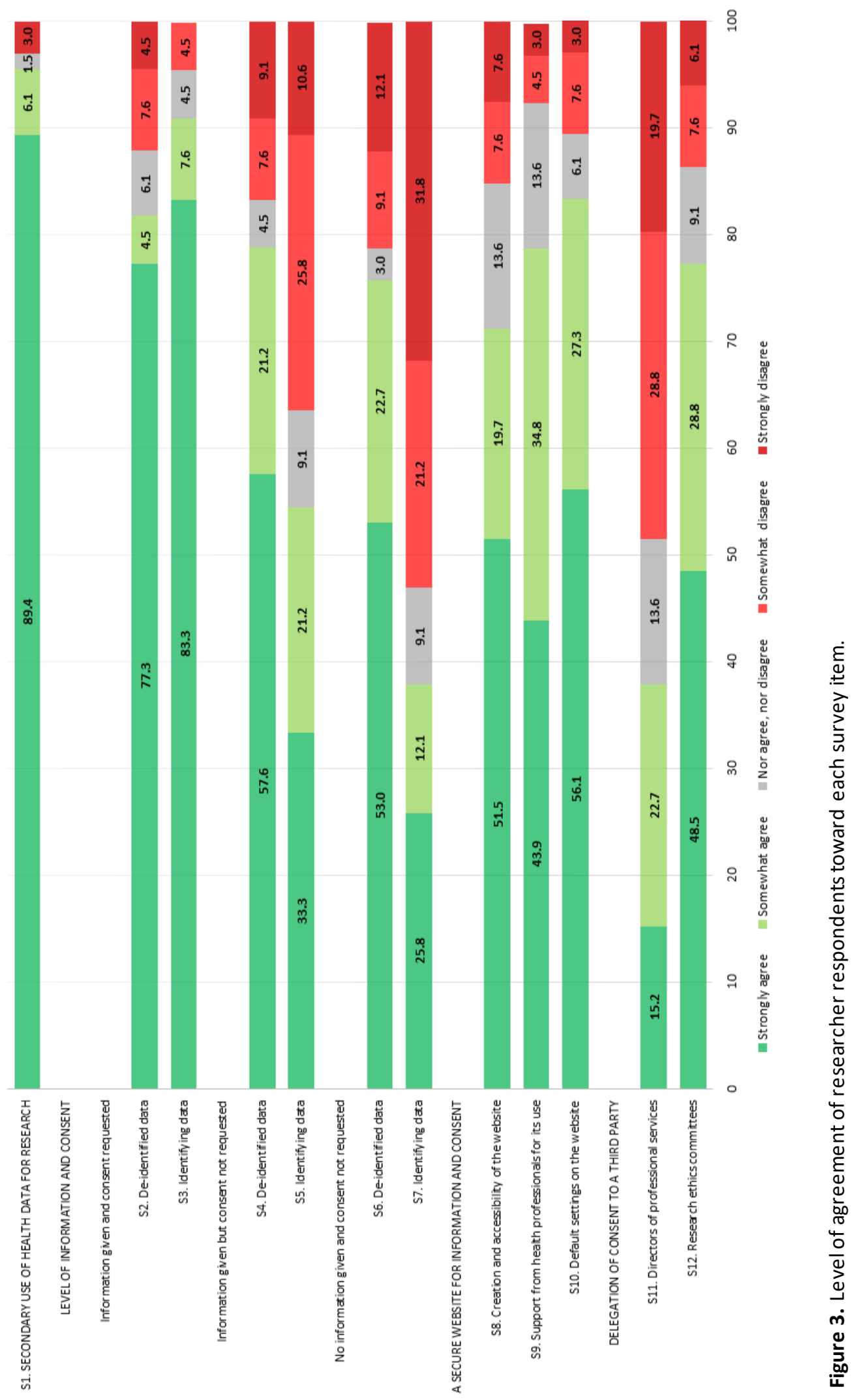




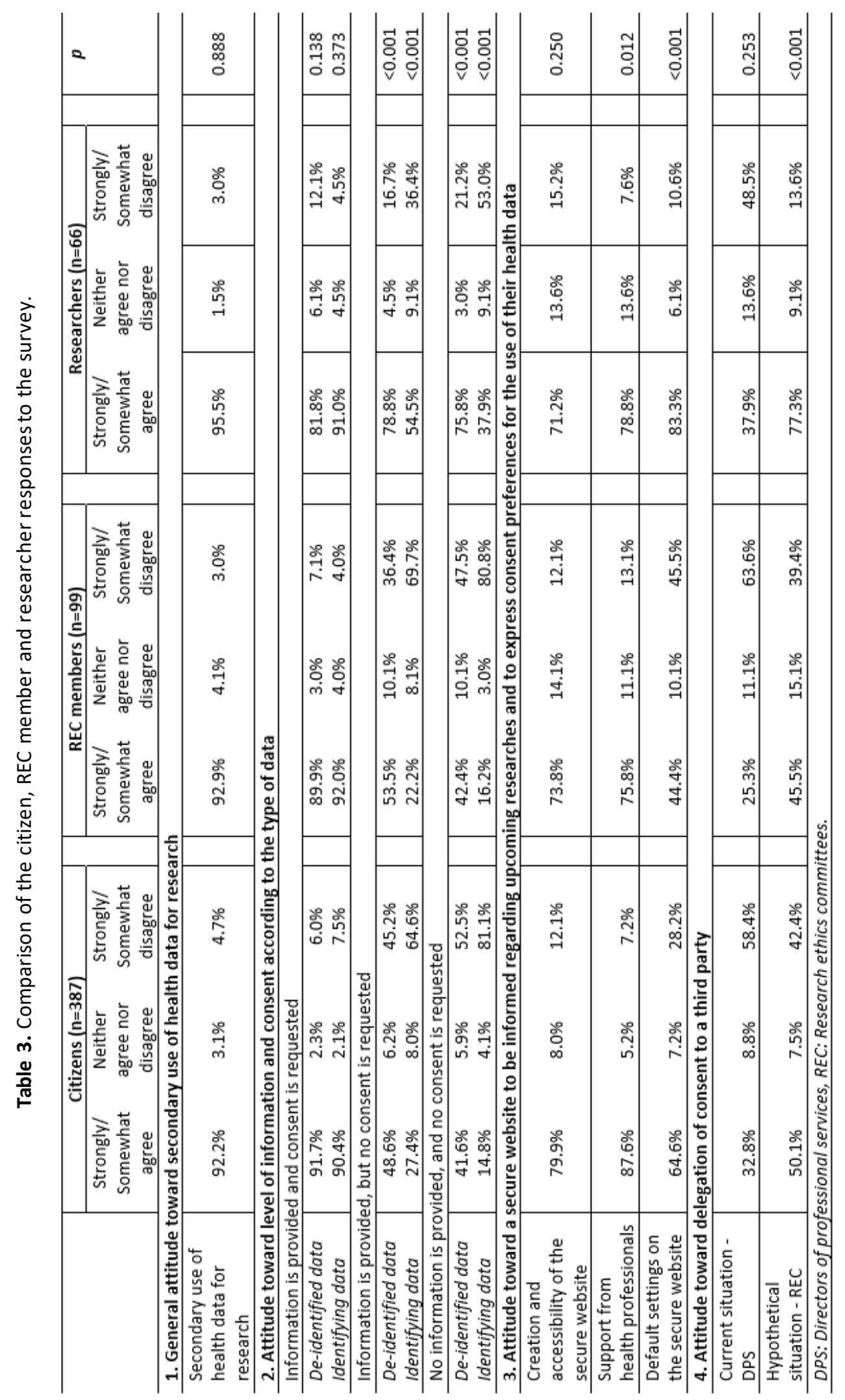


Table 4. Respondents' attitude regarding individual control and common good.

\begin{tabular}{lccc}
\hline $\begin{array}{l}\text { Question: } \\
\text { Which statement is more } \\
\text { important to you? }\end{array}$ & $\begin{array}{c}\text { Individual control: } \\
\text { It is more important that } \\
\text { each citizen may easily } \\
\text { decide whether his or her } \\
\text { health data is used for } \\
\text { research. }\end{array}$ & $\begin{array}{c}\text { Common good: } \\
\text { It is more important that the } \\
\text { citizens' health data be easily } \\
\text { accessible for research. }\end{array}$ & $\begin{array}{c}\text { Refuse to take } \\
\text { position }\end{array}$ \\
\hline Citizens $(n=387)$ & $238(61.5 \%)$ & $145(37.5 \%)$ & $4(1.0 \%)$ \\
\hline REC members $(n=99)$ & $63(63.6 \%)$ & $25(25.3 \%)$ & $11(11.1 \%)$ \\
\hline Researchers $(n=66)$ & $13(19.7 \%)$ & $46(69.7 \%)$ & $7(10.6 \%)$ \\
\hline REC: Research Ethics Committee. & & & \\
\hline
\end{tabular}

\section{Discussion}

This survey had two main objectives: 1) Draw a current portrait of the attitude of Quebec citizens, REC members and researchers regarding the reuse of people's health data for research as well as regarding certain components of a new consent model (meta-consent) better fitting a LHS; 2) Inform and guide a series of focus groups in order to further explore components of the metaconsent model for its eventual implementation in the Quebec LHS platform. Several interesting observations can be made on our results.

First, Quebec citizens strongly support the reuse of their health data for research to enhance knowledge and improve care, which is aligned with similar surveys conducted in another Canadian province (Willison et al., 2007) and in the US (Cho et al., 2015; Kelley et al., 2015; Kim et al., 2015; Kraybill et al., 2016). However, citizens do not seem to have a positive opinion regarding waiving consent. In fact, the vast majority of them supported the reuse of health data (de-identifiedand identifying) in research when two conditions are met: 1) people are informed about the use of their data and 2) consent is requested. Less than half supported the use of health data (deidentified and identifying) if people are informed about the use of their data, but no consent is requested. As presented in our scoping review (Cumyn et al., 2019), the literature on consent in LHSs emphasizes the central role of the information and consent processes in maintaining trust for the use of data for research. In fact, several studies showed that even if there is good acceptance from the public for research with health data, there is a strong desire of transparency and to be asked for permission before using this information. As illustrated in our scoping review, participants expressed a preference for an "ask me each time" model of consent in research (Flory et al., 2016; Kaplan et al., 2016; Kass et al., 2016; Kelley et al., 2015; Nayak et al., 2015; Sugarman 
\& Califf, 2014; Weinfurt et al., 2016; Whicher et al., 2015). However, the study by Cho et al. (2015) noted that when the consent process impacts significantly the feasibility of the research, participants were willing to accept less elaborate approaches. The literature seemed to suggest that when the trade-offs are better understood by participants, what matters most to the public may be being informed directly and retaining some degree of decision-making control (for instance an initial broad consent) (Cho et al., 2015; Willison et al., 2007), which is compatible with a meta-consent model, rather than a more intrusive and cumbersome study-specific consent process (Simon et al., 2011; Thiel et al., 2014; Weinfurt et al., 2016).

It is interesting to note that REC members had opinions more aligned with citizens than with researchers in this survey regarding the use of health data for research without informing and obtaining consent from people for both de-identified and identifying data. This finding is important, as one of the roles of RECs is the protection of research participants. An alignment with citizens' views might be relevant to achieve this objective. Researchers seem to support more strongly the use of de-identified health data without information nor people's consent. As the use of de-identified health data is crucial for certain research projects for which consent cannot always be obtained, this result is not very surprising. Several researchers and ethicists described specific individual consent with opt-in as impractical, as it would be too time consuming and expensive to ask consent from every patient for every research project (Faden et al., 2013; Hoffman \& Podgurski, 2012; Kaplan et al., 2016). Few seem to believe that this approach to consent would fit well the needs of LHSs. Nevertheless, the researchers substantially agree d with a delegated consent model to RECs which might indicate a fair level of trust in RECs by researchers despite having different opinions on some issues.

More citizen respondents agreed with the use of de-identified data than with the use of identifying data if information is not provided and people's consent is not sought ( $41.6 \%$ strongly or somewhat agreed vs $14.8 \%$ ). Similar observations were made in several other studies (Kaplan et al., 2016; Kim et al., 2015; Willison et al., 2007). In their qualitative study, Mayo et al. (2017) showed that participants accept that researchers and hospitals (for most uses) would access their data but would be nervous at the idea that drug companies or insurance companies would have access to "even some of those data". Patients may fear their data could be used for wrongful reasons. For example, they may fear that insurance companies could have access to medical data and deny coverage on this basis. This concern could decrease people's consent to a general use 
of their data, especially their identifying data, for all research purposes. We posit that a lack of information may lead to a magnification of such concerns (Cumyn et al., 2019).

The vast majority of our citizen respondents found it important that a website be created to allow citizens to be notified of the use of their health data for upcoming research projects and to express their consent preferences on this matter. It appears that Quebec citizens would like to have control over their health data and seem to be open to use a web-based platform to manage its use. This finding is aligned with the fundamental principle of the meta-consent model, which is a form of dynamic consent based on a computer-based technology (Ploug \& Holm, 2016). As showed in several studies, dynamic consent appears to promote participation to research by informed and scientifically literate participants (Daniel \& Choquet, 2016) as well as increase participants' trust (Staa et al., 2016; Williams et al., 2015). Many studies considered it to be practical, respectful and supportive of patient autonomy (Angrist \& Jamal, 2015; Shelton, 2011; Williams et al., 2015).

The citizen respondents strongly agreed with the importance of healthcare professionals supporting people in their use of the website and helping them to better understand the different consent choices proposed on the website. These results confirm that the results in our scoping review (Cumyn et al., 2019) seem applicable to the province of Quebec. Indeed, many studies highlighted the important role of the healthcare provider, especially the physicians, in the information and consent processes regarding the participation in research activities. In a study by Kelley et al. (2015), participants strongly prefer a notification or consent process led by their physician rather than by researchers or other clinical staff. In a similar study (Cho et al., 2015), $84.5 \%$ of patients surveyed preferred to be asked permission to participate in the medical records review study by their physician as opposed to by a researcher or research nurse not involved in their care. However, participation of healthcare professionals in a consent process may come at a cost. Further research will be needed to study issues related to practicability, engagement from healthcare professionals and alignment with ethical principles and regulations.

A majority of citizen respondents agreed with the use of people's health data according to default settings that are aligned with current privacy laws. Default settings are one of the six essential elements of a meta-consent model (Ploug \& Holm, 2016). Default settings allow individual control while having the potential to promote research, as individuals can change their settings at any time according to their preferences. As a system of default settings is difficult to understand, 
especially in a survey with little information, it will be important to further explore its understanding in the upcoming focus groups. It seems that no other studies have evaluated attitudes toward this characteristic of meta-consent.

Citizen respondents generally expressed low acceptability regarding the fact that a third party (e.g. a member of the hospital management) could decide on the people's behalf the use of their health data for research purposes. In the same vein, interestingly, REC members and researchers did not wholeheartedly agree either with the delegation of consent to an hospital administrator. This result shows that the public, as well as the other concerned parties, seem to disagree with the models of delegated consent that are applied in some contexts, including in Quebec for retrospective studies with hospital health data. Citizens seem to want to retain control over their health data. The creation of a secure website to allow citizens to have the con trol over their data would built trust: people will be more willing to share their data if they know when they are used, for what purposes and what are the impact of this research on improving care.

\section{Limitations}

Our findings should be interpreted with some caveats in mind. First of all, we are aware of the potential selection bias related to the use of BIP Research sampling frame to generate our study sample and the possible impact of this bias on the generalising of our findings to the entire province of Quebec. Also, we are aware that telephone surveys have their own challenges such as nonresponse bias, particularly that response rates have been on a precipitous decline (Curtin et al., 2005). However, our results still provide a good starting point for analysing the opinions of Quebec citizens on issues surrounding consent for the use of health data in research and are part of a larger sequential mixed-methods study. Also, the impact of data access is likely to be stronger for older citizens given the higher probability that they receive health care from a health organisation, and so that health data is generated.

Secondly, the use of closed-ended questions limits the interpretation of the results, since it was not possible for respondents to qualify their answers. Because of that, our results should be interpreted with some caution considering that they provide only a partial picture of the attitudes of the various concerned parties. For example, with regards to the default settings, it was difficult to present the possible nuances associated with them. Some respondents might have considered that these settings would automatically permit use of data when in fact, a default setting could 
be an "ask me each time" setting. For that reason, further research following a qualitative methodology will be necessary to explore this perception in depth.

Thirdly, contextualisation within the LHS framework was not done explicitly in the survey because this concept is complex and unknown to a majority of study participants. It is possible that answers to statements could have varied if specific contexts had been proposed. Nevertheless, it was important to have such a survey to characterise the current situation and opinions of the participants in order to inform future changes. The survey is part of a larger sequential mixedmethods study and will be used to inform the content of focus groups in which the LHS context will be made explicit.

Fourthly, we asked for respondents' opinion regarding research in general. Their attitudes might have been different if we had presented them more specific research scenarios (e.g., academic research vs. private research). Since this is more difficult to assess in a survey, we will explore these concepts in the focus groups.

Finally, we are aware that our survey did not capture all aspects related to the meta-consent model and does not provide a comprehensive picture of the opinions of citizens, researchers and REC members on this topic.

\section{Best Practices}

Based on our findings, Quebec citizens largely support the use of their health data for research to advance knowledge and improve care, but they value transparency and they place a high importance on personal control on the use of their health data for research. In that regard, our results suggest that they would support the development of a web-based platform to be informed about incoming research projects and to express their consent preferences regarding the use of their health data.

For these reasons, policy makers need to consider the public opinion regarding the use of their health data for research. In addition, they need to take into consideration the evolution of our healthcare system into a LHS. Accordingly, changes to current consent and data access models will need to be carefully considered.

\section{Research Agenda}


This survey is part of a larger project that explores public perspectives on alternate approaches to the current consent models in Quebec to take into consideration the unique features of a LHS. This survey as well as the focus groups will lead to key recommendations that will support and guide decision makers at local and provincial levels in future decisions on this issue. The ultimate goal is to review and adjust the model of consent in Quebec, where needed. This model will need to ensure that citizens have the opportunity to be better informed about incoming researches with their health data and have their say, when possible, in the use of their information.

\section{Educational Implications}

A dialogue must be initiated with front-line professionals to explore their perception about being actively involved in the process of informing their patients about research with health data and in supporting their decision-making regarding consent for the use of their own health data for research. We need to define their roles and responsibilities in the possible deployment of metaconsent in Quebec.

\section{Acknowledgements}

The authors thank Paula Bush for providing her expertise on survey design, AndréeAnne Beaudoin for her support in data analysis, Nissrine Safa as a scientific research assistant in the project as well as Virginie Duceppe-Lamarre for the linguistic review of the survey and the manuscript.

\section{Declaration of conflicting interests}

Annabelle Cumyn is REC Chair. However, the project was reviewed and approved by a separate REC. The authors declared no other potential conflicts of interest with respect to the research, authorship, and/or publication of this article.

\section{Funding}

This study was supported by the QuebecSPOR Support Unit, an initiative funded by the Canadian Institutes of Health Research (CIHR), the Ministère de la santé et des services sociaux du Québec, and the Fonds de recherche du Québec - Santé (co-PI - Jean-François Ethier). These three funders were not involved in the design, data collection, the interpretation or the publication of the study. 


\section{References}

Angrist, M., \& Jamal, L. (2015). Living laboratory: Whole-genome sequencing as a learning healthcare enterprise. Clinical Genetics, 87(4), 311-318.

https://doi.org/10.1111/cge.12461

BIP Research. (2015). BIP Research. https://www. bipresearch.com/

Budrionis, A., \& Bellika, J. G. (2016). The Learning Healthcare System: Where are we now? A systematic review. Journal of Biomedical Informatics, 64, 87-92. https://doi.org/10.1016/j.jbi.2016.09.018

Cho, M. K., Magnus, D., Constantine, M., Lee, S. S.-J., Kelley, M., Alessi, S., Korngiebel, D., James, C., Kuwana, E., Gallagher, T. H., Diekema, D., Capron, A. M., Joffe, S., \& Wilfond, B. S. (2015). Attitudes Toward Risk and Informed Consent for Research on Medical Practices: A Cross-sectional Survey. Annals of Internal Medicine, 162(10), 690-696. https://doi.org/10.7326/M15-0166

Cumyn, A., Barton, A., Dault, R., Cloutier, A.-M., Jalbert, R., \& Ethier, J.-F. (2019). Informed consent within a learning health system: A scoping review. Learning Health Systems, n/a(n/a), e10206. https://doi.org/10.1002//rh2.10206

Curtin, R., Presser, S., \& Singer, E. (2005). Changes in Telephone Survey Nonresponse over the Past QuarterCentury. https://doi.org/10.1093/POQ/NFI002

Daniel, C., \& Choquet, R. (2016). Clinical Research Informatics Contributions from 2015. Yearbook of Medical Informatics, 1, 219-223. https://doi.org/10.15265/IY-2016-044

Delaney, B. C., Ethier, J.-F., Curcin, V., Corrigan, D., \& Friedman, C. (2013). International perspectives on the digital infrastructure for The Learning Healthcare System. 2013. https://doi.org/10.13140/2.1.3015.6165 
Faden, R., Kass, N., Whicher, D., Stewart, W., \& Tunis, S. (2013). Ethics and informed consent for comparative effectiveness research with prospective electronic clinical data. Medical Care, 51(8 Suppl 3), S53-57. https://doi.org/10.1097/MLR.0b013e31829b1e4b

Faden, R. R., Kass, N. E., Goodman, S. N., Pronovost, P., Tunis, S., \& Beauchamp, T. L. (2013). An ethics framework for a learning health care system: A departure from traditional research ethics and clinical ethics. Hastings Center Report, 43(s1), S16-S27.

Flory, J. H., Mushlin, A. I., \& Goodman, Z. I. (2016). Proposals to Conduct Randomized Controlled Trials Without Informed Consent: A Narrative Review. Journalof General Internal Medicine, 31(12), 1511-1518. https://doi.org/10.1007/s11606-016-3780-5

Fox, S. (2011). The Social Life of Health Information, 2011. 45.

Gouvernement du Québec. (2020a). Centres et réseaux_Fonds Santé. http://www.frqs.gouv.qc.ca/la-recherche/la-recherche-financee-par-le-frqs/centres-etreseaux?field=all\&grouptype=1\&submit=Rechercher

Gouvernement du Québec. (2020b). Répertoire des ressources du réseau de la santé et des services sociaux en éthique et autorisation des recherches-Aperçu de l'éthique en santé et services sociaux - Professionnels de la santé-MSSS. https://www.msss.gouv.qc.ca/professionnels/ethique/ethique-en-sante-et-servicessociaux/repertoires/

Government of Canada. (2019, April 1). Tri-Council Policy Statement:Ethical Conductfor Research Involving Humans - TCPS 2 (2018). https://ethics.gc.ca/eng/policypolitique_tcps2-eptc2_2018.html Grajales, F., Clifford, D., Loupos, P., Okun, S., Quattrone, P., Simon, M., Wicks, P., \& Henderson, D. (2014). Social Networking Sites and the Continuously Learning Health System: A Survey. NAM Perspectives, 4(1). https://doi.org/10.31478/201401d 
GRIIS. (2019). PARS3. GRIIS. https://griis.ca/en/solutions/pars3/

Hoffman, S., \& Podgurski, A. (2012). Balancing Privacy, Autonomy, and Scientific Needs in Electronic Health Records Research (SSRN Scholarly Paper ID 1923187). Social Science Research Network. https://papers.ssrn.com/abstract=1923187

Institut de la statistique du Québec. (2018). Le bilan démographique du Québec. Édition 2018. 174.

Institut de la Statistique du Québec. (2019). Répartition de la population de 25 à 64 ans selon le plus haut niveau de scolarité atteint, la région administrative, l'âge et le sexe, Québec et région administratives, 1990 à 2018.

http://www.stat.gouv.qc.ca/statistiques/education/niveau-scolarite/repartition-scol-rasexe-age.html\#tri_tertr=50040000000000000\&tri_sexe=1\&tri_age=365\&tri_stat=8403 Kaplan, S. H., Gombosev, A., Fireman, S., Sabin, J., Heim, L., Shimelman, L., Kaganov, R., O sann, K. E., Tjoa, T., \& Huang, S. S. (2016). The patient's perspective on the need for informed consent for minimal risk studies: Development of a survey-based measure. $A J O B$ Empirical Bioethics, 7(2), 116-124. https://doi.org/10.1080/23294515.2016.1161672 Karbwang, J., Koonrungsesomboon, N., Torres, C. E., Jimenez, E. B., Kaur, G., Mathur, R., Sholikhah, E. N., Wanigatunge, C., Wong, C.-S., Yimtae, K., Abdul Malek, M., Ahamad Fouzi, L., Ali, A., Chan, B. Z., Chandratilake, M., Chiew, S. C., Chin, M. Y. C., Gamage, M., Gitek, I., ... FERCAP Multi-Country Research Team. (2018). What information and the extent of information research participants need in informed consent forms: A multicountry survey. BMCMedical Ethics, 19(1), 79. https://doi.org/10.1186/s12910-0180318-x

Kass, N., Faden, R., Fabi, R. E., Morain, S., Hallez, K., Whicher, D., Tunis, S., Moloney, R., Messner, D., \& Pitcavage, J. (2016). Alternative consent models for comparative effectiveness 
studies: Views of patients from two institutions. AJOB Empirical Bioethics, 7(2), 92-105. https://doi.org/10.1080/23294515.2016.1156188

Kelley, M., James, C., Alessi Kraft, S., Korngiebel, D., Wijangco, I., Rosenthal, E., Joffe, S., Cho, M. K., Wilfond, B., \& Lee, S. S.-J. (2015). Patient Perspectives on the Learning Health System: The Importance of Trust and Shared Decision Making. The American Journal of Bioethics: AJOB, 15(9), 4-17. https://doi.org/10.1080/15265161.2015.1062163

Kim, K. K., Joseph, J. G., \& Ohno-Machado, L. (2015). Comparison of consumers' views on electronic data sharing for healthcare and research. Journalof the American Medical Informatics Association : JAMIA, 22(4), 821. https://doi.org/10.1093/jamia/ocv014

Kraybill, A., Dember, L. M., Joffe, S., Karlawish, J., Ellenberg, S. S., Madden, V., \& Halpern, S. D. (2016). Patient and Physician Views about Protocolized Dialysis Treatment in Randomized Trials and Clinical Care. AJOB Empirical Bioethics, 7(2), 106-115. https://doi.org/10.1080/23294515.2015.1111272

Lessard, L., Michalowski, W., Fung-Kee-Fung, M., Jones, L., \& Grudniewicz, A. (2017). Architectural frameworks: Defining the structures for implementing learning health systems. Implementation Science :IS, 12. https://doi.org/10.1186/s13012-017-0607-7

Mayo, R. M., Summey, J. F., Williams, J. E., Spence, R. A., Kim, S., \& Jagsi, R. (2017). Qualitative Study of Oncologists' Views on the CancerLinQ Rapid Learning System. Journal of Oncology Practice, 13(3), e176-e184. https://doi.org/10.1200/JOP.2016.016816

Nayak, R. K., Wendler, D., Miller, F. G., \& Kim, S. Y. H. (2015). Pragmatic Randomized Trials Without Standard Informed Consent?: A National Survey. Annals of Internal Medicine, 163(5), 356-364. https://doi.org/10.7326/M15-0817

Park, J. W., \& Jung, M. S. (2009). A Note on Determination of Sample Size for a Likert Scale. Communications for Statistical Applications and Methods, 16(4), 669-673. 
Ploug, T., \& Holm, S. (2016). Meta Consent - A Flexible Solution to the Problem of Secondary Use of Health Data. Bioethics, 30(9), 721-732. https://doi.org/10.1111/bioe.12286

Ploug, T., \& Holm, S. (2017). Informed consent and registry-based research-the case of the Danish circumcision registry. BMCMedical Ethics, 18(1), 1-10.

Shelton, R. H. (2011). Electronic consent channels: Preserving patient privacy without handcuffing researchers. Science Translational Medicine, 3(69), $69 \mathrm{~cm} 4$. https://doi.org/10.1126/scitransImed.3002037

Simon, C. M., L’Heureux, J., Murray, J. C., Winokur, P., Weiner, G., Newbury, E., Shinkunas, L., \& Zimmerman, B. (2011). Active choice but not too active: Public perspectives on biobank consent models. Genetics in Medicine : OfficialJournal of the American College of Medical Genetics, 13(9), 821-831. https://doi.org/10.1097/GIM.0b013e31821d2f88

Staa, T.-P. van, Goldacre, B., Buchan, I., \& Smeeth, L. (2016). Big health data: The need to earn public trust. BMJ, 354. https://doi.org/10.1136/bmj.i3636

Sugarman, J., \& Califf, R. M. (2014). Ethics and regulatory complexities for pragmatic clinical trials. JAMA, 311(23), 2381-2382. https://doi.org/10.1001/jama.2014.4164

The learning healthcare project. (2020). Learning Health Care. http://www.learninghealthcareproject.org/section/background/learning-healthcaresystem

Thiel, D. B., Platt, T., Platt, J., King, S. B., \& Kardia, S. L. R. (2014). Community perspectives on public health biobanking: An analysis of community meetings on the Michigan BioTrust for Health. Journal of Community Genetics, 5(2), 125-138. https://doi.org/10.1007/s12687-013-0162-0

Weinfurt, K. P., Bollinger, J. M., Brelsford, K. M., Crayton, T. J., Topazian, R. J., Kass, N. E., Beskow, L. M., \& Sugarman, J. (2016). Patients' Views Concerning Research on Medical 
Practices: Implications for Consent. AJOB Empirical Bioethics, 7(2), 76-91.

https://doi.org/10.1080/23294515.2015.1117536

Whicher, D., Kass, N., \& Faden, R. (2015). Stakeholders' Views of Alternatives to Prospective Informed Consent for Minimal-Risk Pragmatic Comparative Effectiveness Trials. The Journal of Law, Medicine \& Ethics: A Journal of the American Society of Law, Medicine \& Ethics, 43(2), 397-409. https://doi.org/10.1111/jlme.12256

Williams, H., Spencer, K., Sanders, C., Lund, D., Whitley, E. A., Kaye, J., \& Dixon, W. G. (2015). Dynamic Consent: A Possible Solution to Improve Patient Confidence and Trust in How Electronic Patient Records Are Used in Medical Research. JMIR Medical Informatics, 3(1). https://doi.org/10.2196/medinform.3525

Willison, D. J., Schwartz, L., Abelson, J., Charles, C., Swinton, M., Northrup, D., \& Thabane, L. (2007). Alternatives to Project-specific Consent for Access to Personal Information for Health Research: What Is the Opinion of the Canadian Public? Journal of the American Medical Informatics Association :JAMIA, 14(6), 706-712.

https://doi.org/10.1197/jamia.M2457 